LA-UR. 93-547

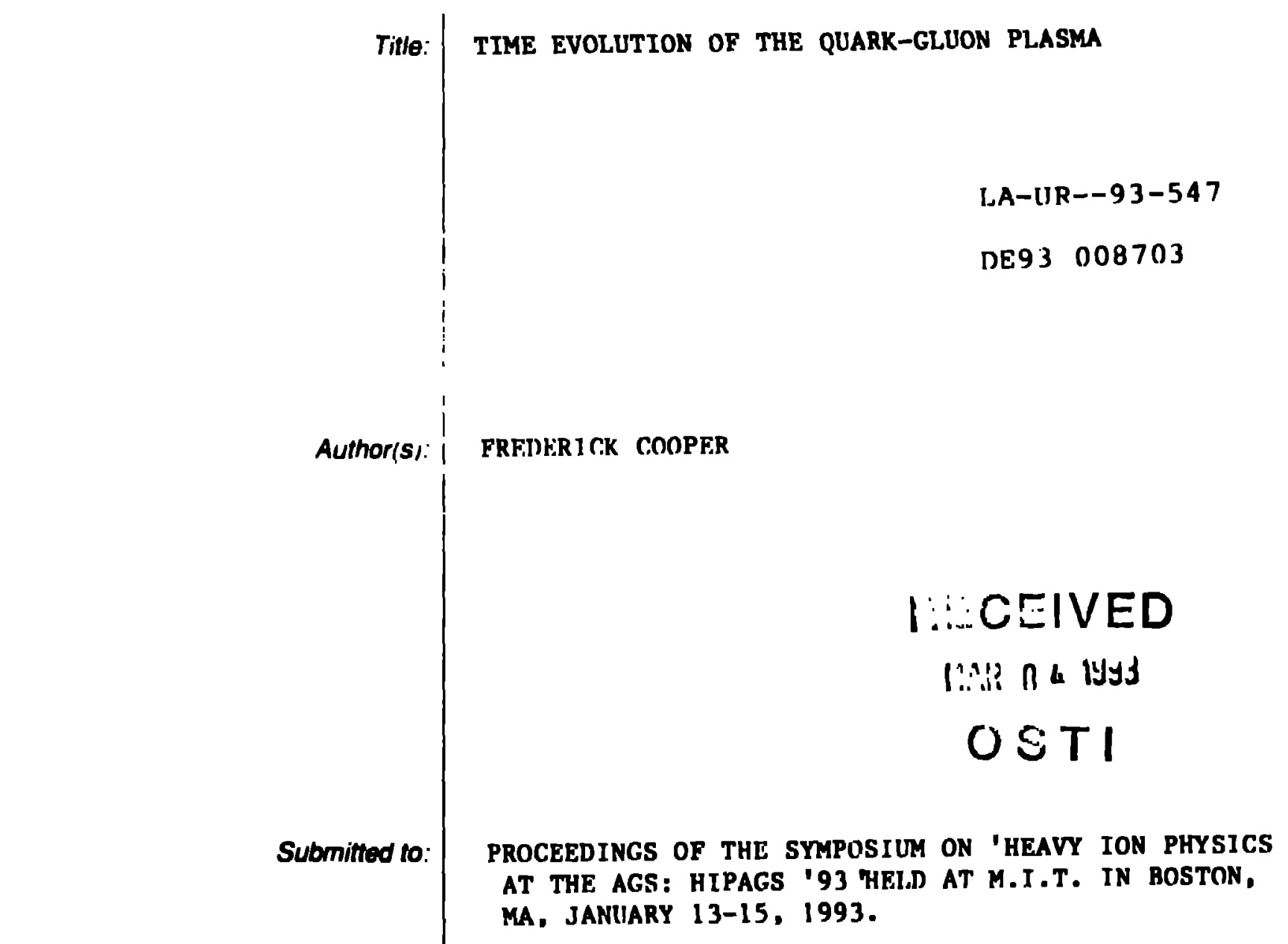

DISTRIBUTION OF THIS DOCUMENT IS UNLIMUTEO

$y^{22}$

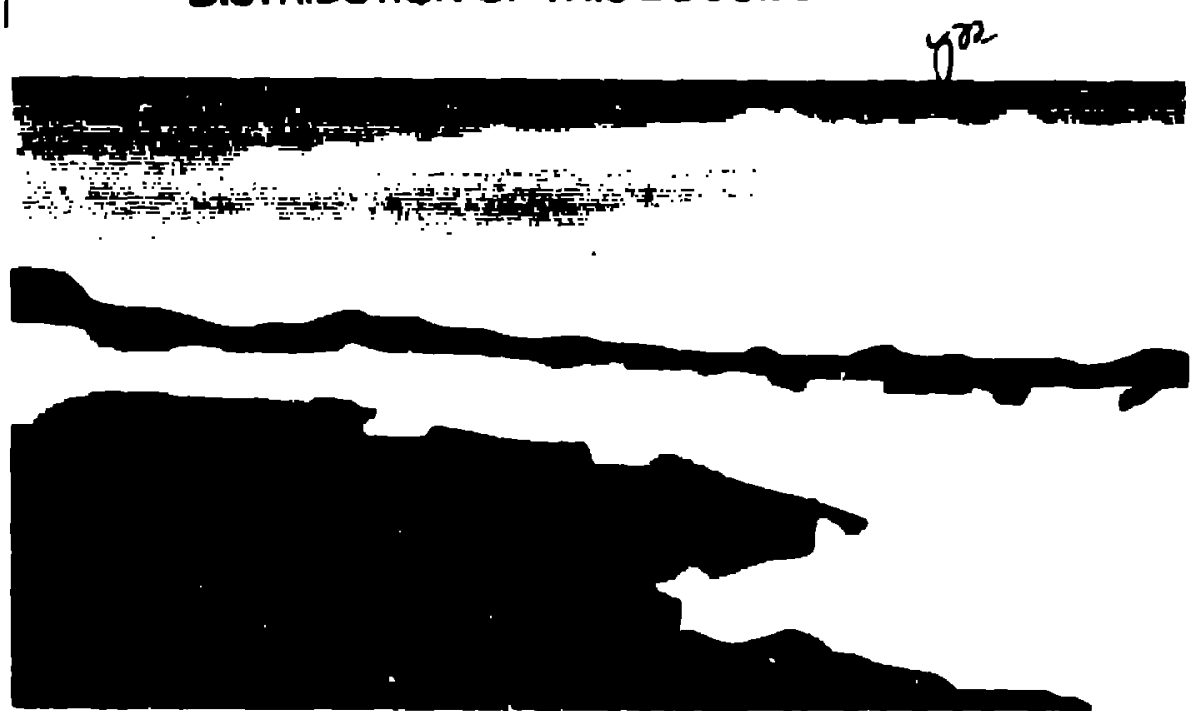

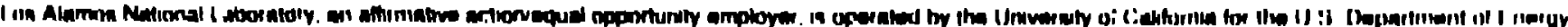

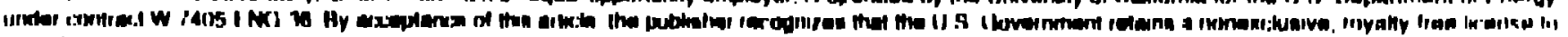

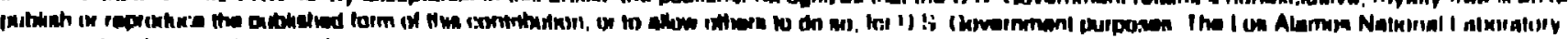

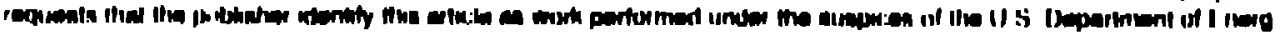




\title{
TIME EVOLUTION OF THE QUARK-GLUON PLASMA
}

\author{
FRED COOPER \\ Theortical Division, Los Alamos National Laboratory, \\ Los Alamos, NA1875 85 \\ and \\ Department of Physics, Universily of New Ilampshire, \\ Durham, NII 03824
}

\begin{abstract}
ABSTHACT
We review progress in our molerstanding the production and time evolution of the quark gluon plasma startitig with boost invariant initial conditions in a field theory model based on the Schwinger merlianism of particle production via tunnelling.
\end{abstract}

\section{Introduction}

The present theory of Strong Interactions, namely $Q$ uantum ('hromodynamics $\left(Q\left({ }^{\prime}\right)\right)$ has the property that the glarks, which are the building blocks of the protons and neulrons, are confined and cannot be liberated. 'Thus one ran infer the properties of tion quarks only' indirectly using high energy probes which probe the short destant interactions of the guarks. In recent years. as a result of lattice gauge therory calculations, it was found that at high

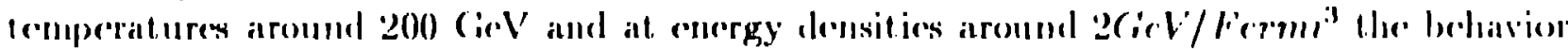
of $Q(')$ changes and there appears to be a first order phase transition from lhe phase where

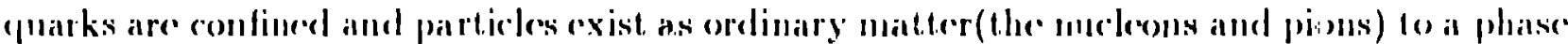
where there are guaths and gluons that are interacling weakly as in a weakly interacting, fas lhat is the quark-gluon plasmatia plaser.

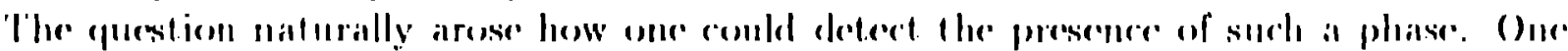

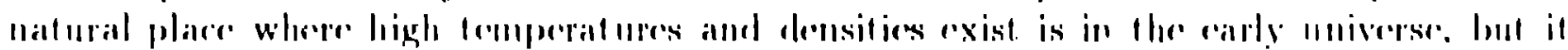

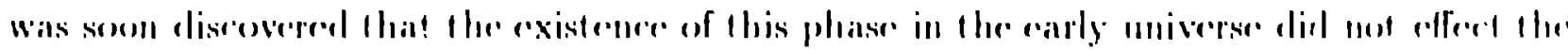

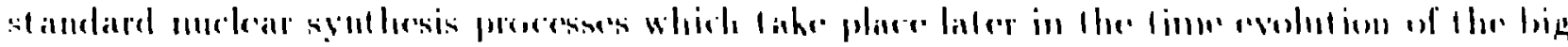

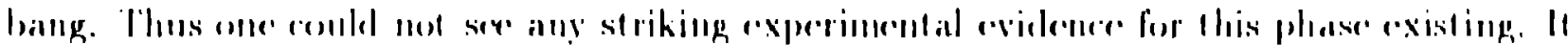

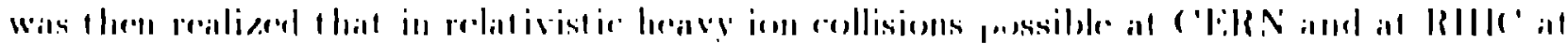

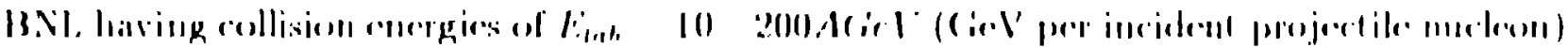

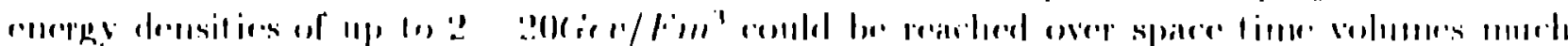

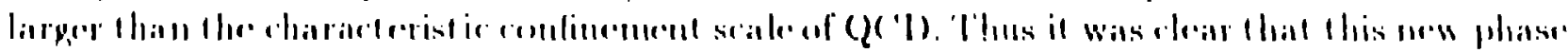

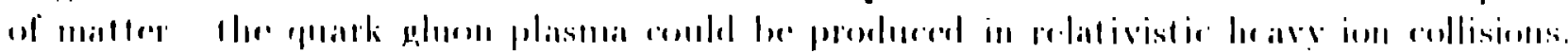

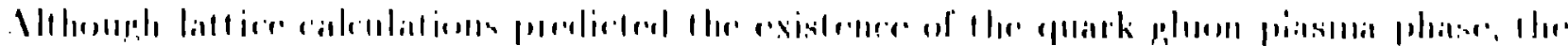

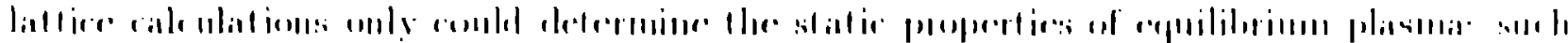

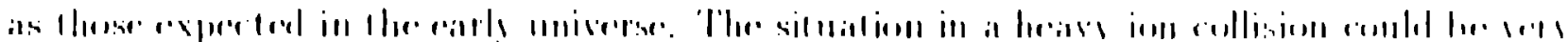

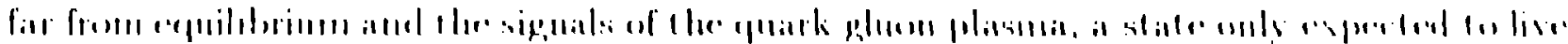


for $10^{-22}$ sec before turning into ordinary hadronic matter, might easily be washed ont by interactions laking place in the ordinary matter phase. 'Thus one needs to look for processes taking piace from a non-equilibrium plasma which are not affected by future interactions. One such process is the production of weakly interacting lepton pairs which escape the. plasma without interacting strongly with the hadronic matter.

In order to determine the spectra of such processes and see if the production of weakly interacting particles coming from this phase is different than that coming from orciinary matter one needs to know the time evolution of the quark-gluon plasna and the distribution of quarks and ant iquarks as a function of time since these are the source of the dileptons. 'This required a new formaiism which allowed one to track the time cvolution of a field theory starting from initial data. The need for such a formalism arose in a previous situation recently in connection with the time evolution of the early unis rse namely the inflationary picture of the early universe. ${ }^{1,2}$ The formalism for doing this latter problem was developerd ${ }^{3}$ and then applied to the problem of pair production by strong electric fields. 'These original simulations of pair production ${ }^{4}$ became the prototype for a more serious investigation of the time erolution of the ciuark-gluon plasma. What does relativistic heavy ion collision look like": 'The strong forces between hadrons are mediated by colored gluons, and as a result of rxchanging gluons, the initially colorless hadrons become charged witl: opposite colors and get separated quickly since the hadrons move away from each other near the spered of light ". Thus one has a situation resembling 1 wo charged plates moving away from each other with a constant colored electric field between them. When the energy density in the field is large enough so that the work done by the field at a hadronic distance is equal to the rest energy of a quark anti-guark pair, then the "vacuum" becomes unstable to the prosluction of this pair. 'The criteria for this to happen is:

$$
y l\left(h / m C^{\circ}\right)>2 m r^{2}
$$

where ga is the colere charge, li the color electrie field and h/me is the llatural size the

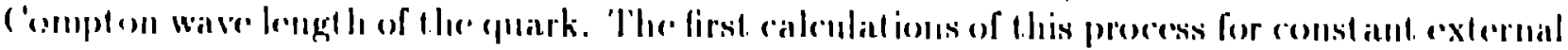

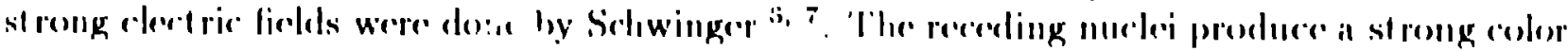

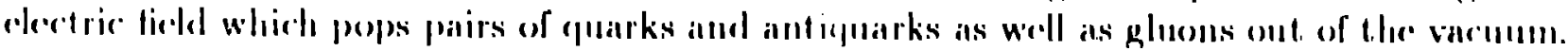
A plasma of plateks and glue moving in and interacting with the original time varying color

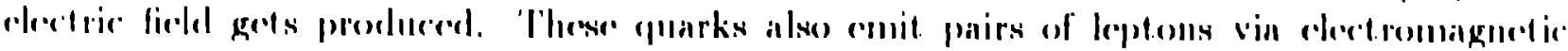

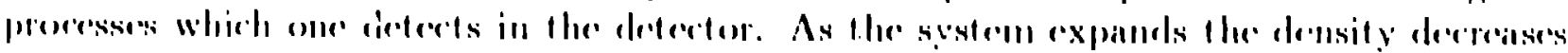

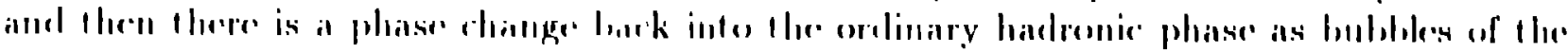

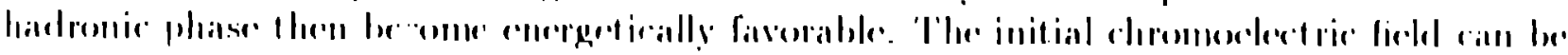

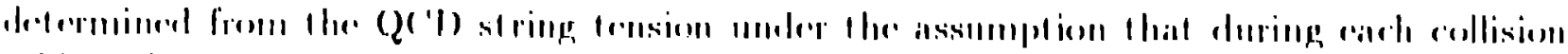

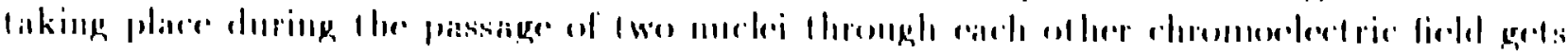

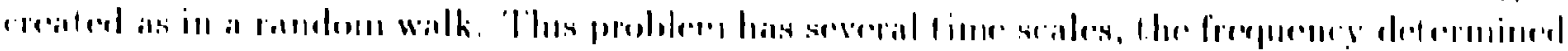

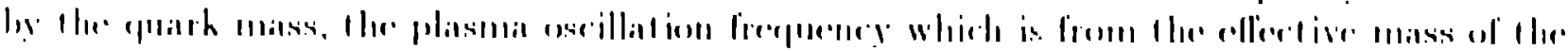

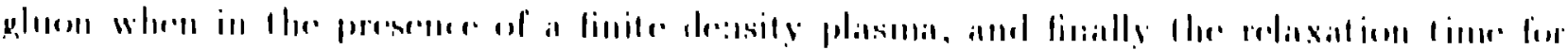

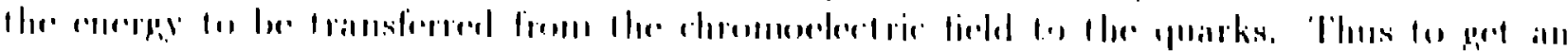

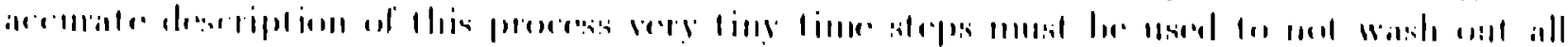

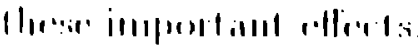

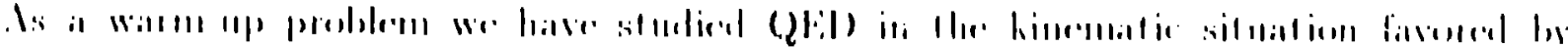

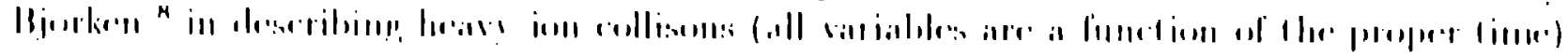


and determined the time evolution of the quarks in the background field covolving in proper time $\tau$. In this simplified model we have been able to check many of the assumptions of more phenomenolegical pictures of the time evolution of the quark-ghuon plasma. We have compare: wur exact calculations with semi-classical transport models which assume the mechanism of pair production described above as a source term for the time evolution of a single particle distribution function similar to that used in ordinary plasma physics ${ }^{9}, 10$, ${ }^{11}$, as woll as tested several assumptions of hydrodynamic models ${ }^{12,13,8}$ which assume linit. global macroscopic variables such as energy density and pressure can be used to study the time evolution of the plasma as one does in ordinary relativistic fuid flows.

What we found was that previous transport morlels had ignored a hasic important fact the Pauli-exclusion principle which does not allow two quarks to be in the same state. IVhen we includerl this fact we found that at least for the case where we could ignore the gintut um nuctuations of the colored ficlds, that the modified transport theory agreed well with a coarse grained average (in both time and momentum space) of our field theory calculation. IVe also were able to verify some of the assumptions of hydrodynamic models, even thoigh for our calculation we were far from the modynamic equilibrium. By stuelying the microscopic analogues of energy density and pressure, we were alsi" to verify that particle production rates could be determined from the hydrodynamic energy flows as assumed in hyctrodyuamie modrls.

We are now entering the next phase of our approach. In our previous calculation we ignored the guantum fluctuations of the colored electric fields as well as the scat tering among the quarks and anti-quarks. Wo have drveloperl a systematic method of calculat ing these efferts. We also have developed the formalism for st udying the production and lime coulution of the purely gluonic plasma, where the glue is treated in a mean fiedd approximation. Numerical simulations of thewe latter two problems are just begimning.

Drtails of these carlier calculations as well as time coolution results for the elecetric field.

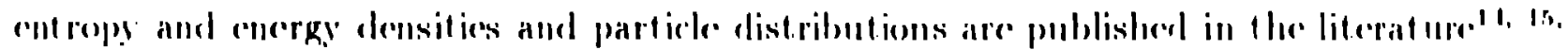
$11 i, 17$

\section{Arknowledgements}

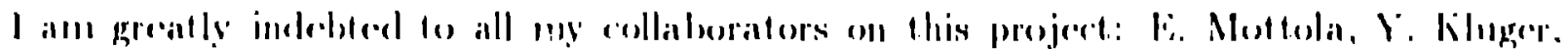

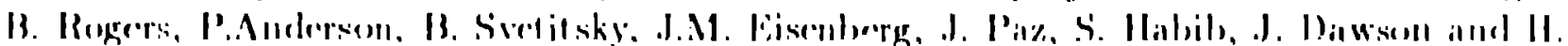
Siliepilirl.

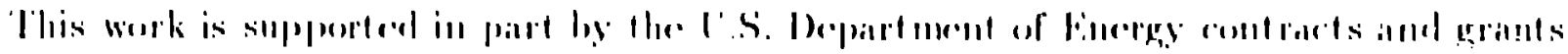
wilh I.INI, anl I'XIl.

\section{Heforencens}

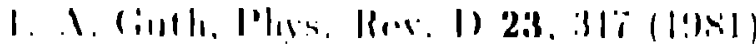

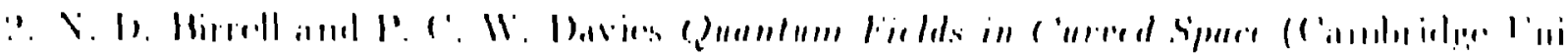

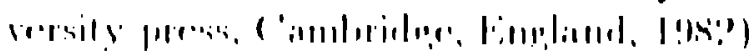

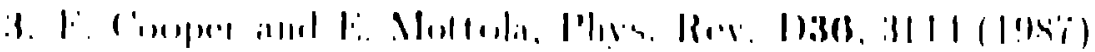

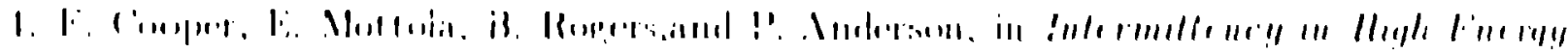

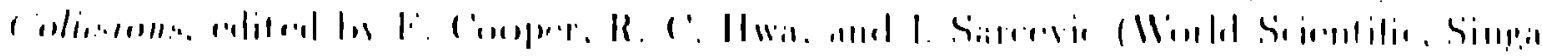

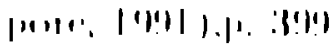


5. T. S. Biró, H. B. Nielsen, and J. Knoll, Nucl. Phys. B245, 449 (1984)

6. F. Sauter, Z. Phys. 69, 742 (1931)

7. J. Schwinger, Phys. Rev. Lett. 82, 664 (1951)

8. J. D. Bjorken, Phys. Rev. D27, 140 (1983)

9. K. Kajantie and 'T. Matsui, Phys. Lett 164B, 373 (1985)

10. A. Bialas and W. Czyż, Acta Phys. Pol. B iт, 635 (1986)

11. G. Gatoff, A. K. Kerman, and T. Matsui, Phys. Rev. D 36

12. L. D. Landau, Izv. Akd. Nauk. SSSIR 17. 51 (1953), 114 (1987)

13. F. Cooper, G. Frye and E. Schonberg, Phys. Rev. D11, $192(1975)$

1.1. F. Cooper and E. Mottola, Phys. Rev. D 40, 156 (1989)

15. Y. Kluger, J. M. Pisonberg, B. Svetitsky, F. Cooper, and E. Mottola, Pliys. Rev. Iett. 67, $2427(1991)$

16. Y. Kluger, J. M. Fisenberg, B. Svetitsky, F. Cooper, and li. Mottola, Phys.Rev. D45, $16.59(1992)$

17. F. Cooper, J. M. lisenberg,Y. K'luger, B. Svetitsky, and E. Mottola, submitted to Phys. Rov. I).

\title{
IMISTI.AIMEK
}

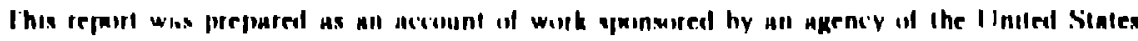

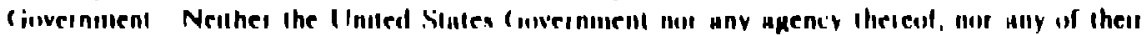

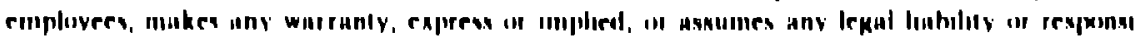

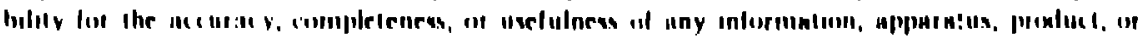

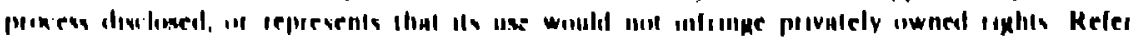

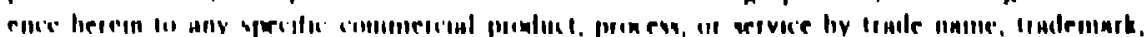

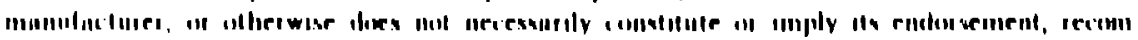

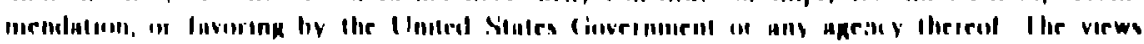

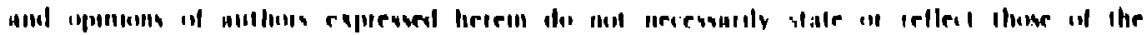

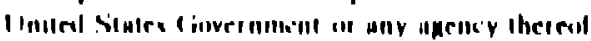

